\section{THE TECHNIQUE OF REMOVING THE AP- PENDIX VERMIFORMIS CECI; REPORT OF ONE HUNDRED CASES.}

Read in the Section on surgery and Anatomy, at the Forty-seventh Annual Meeting of the American Medical Association, held at Atlauta, Ga., Nay 5-S, 1896.

BY M. M. JOHNSON, M.D. HARTFORD, CONN.

Intestinal surgery has, within the last few years, received a long-needed attention. The correct diagnosis of the diseased condition of the appendix, and its operative treatment, has demonstrated the great possibilities of intestinal surgery. As a result we are now saving a class of patients which we have been unnecessarily burying all these many years. As there is a great diversity of opinion as to operative technique it affords a fertile field for our careful consideration.

Prepuration for the Operation.-The patient should receive a general bath in which be is thoroughly washed with a flesh brush and green soap and water, as hot as can be comfortably borne. The abdomen and pubes are shaved, and a green soap poultice applied from two to six hours, followed by a washing with sterilized water, to remove the epidermis which has been acted on by the soap. A towel, wet with 1 to 1,000 solution of bichlorid, is applied until the time of the operation. When upon the table the skin is bathed with ether and alcohol, and covered with a sterilized towel. Patient is now ready for the opertion.

The nails of the operator and the assistant should be short and smooth, their hands and forearms should be washed with soap and water, and with a brush for five minutes. After rinsing with sterilized water, they are immersed in 1 to 1,000 bichlorid solution for ten minutes. This method of hand disinfection has stood the test of bacteriologic examinations. When a more hasty preparation is required, permanganate of potash and oxalic acid are used, according to Holsted.

All dust in the operating room should be removed with a cloth wet with bichlorid solution. As operations frequently have to be performed in an uncleanly house, all dust and dirt in the room, on the furniture, etc., should not be disturbed before the operation. Place a washtub in the middle of the room, and pour boiling water into it. The steam will soon moisten the dust so it will fall to the floor, and the germs with it. You have then a pure atmosphere in which to operate.

Instruments should be sterilized by boiling. Instrument trays should be placed in boiling water, or filled with 5 per cent. solution of carbolic acid. Sponges should be discarded. Sterilized gauze is much safer. All dressings should be wrapped in sterilized towels ready for use when wanted. Care in these minor details is all important.

The Incision.-Surgical opinion as to the length of the incision varies from one and one-half inch to seven or more inches. When we take into consideration the future welfare of the patient, a condition which can not be overlooked by the surgeon, whether the patient be a manual laborer or a brain worker, in either case he is entitled to as firm and sound abdominal wall as can be secured. The shorter the incision, the shorter will be the time the patient must remain in bed, and the more secure will the abdominal walls be. The much criticised statement of Dr. Morris, "An inch and a half a week and a week and a half," contains a sound surgical principle.
While we know that a seven inch incision will unite: as soon as an inch and a half, we also know that, with a short incision, the patient will be in a safer condition to leave his bed in from ten to fourteen days, than the one with a seven inch incision will be in six weeks. In fact, with a long incision, it is a question if the patient will ever be secure from a ventral hernia.

Another very important point is, that the intestines should be manipulated as little as possible.

The shock to the patient and the severity of the colicky stage following the operation, is in proportion to the degree of manipulation to which the intestines are subjected. With a long incision, the escaping intestines protected with a towel are exposed to the air, oftentimes becoming more or less chilled. Much manipulation is required to return and retain them within the abdominal walls. The chance for sepsis and the looping or twisting of the intestines is also great.

In chronic recurrent cases, the writer makes an incision one and one-half inches long directly over the appendix, which has been previously located by palpation, and sufficiently oblique to follow the fibers of the external oblique aponeurosis. When the peritoneum is reached, a catgut suture is passed through it at the upper angle of the incision, before it is cut through. This prevents its retracting and is of material aid in stitching the edges of the peritoneum. This is what Dr. Morris calls his "guy line" device. In complicated cases, the length of the incision must be determined by the room required. I find it best to commence with a short incision and then enlarge as it is necessary.

Method and Complications in Finding the Appendix.-The incision made, the problem of finding the appendix presents itself. Passing the thumb and index finger through the small incision, one quite readily seizes the colon, which is distinguished by a longitudinal muscular ribbon, which is drawn through the incision and returned to prevent exposure, until the appendix is brought into view. The meso-appendix is ligated and incised. When the appendix is thus readily found, the problem is solved.

The position of the appendix is difficult to define. It may be attached to an old hernia sack, to the right ovary or the under surface of the liver. This latter condition the writer found in his 25 th case. The work was much simplified by producing peristalsis by Morris's method of applying a minute quantity of sodium chlorid to the colon, which causes the peristaltic wave to run toward the appendix. Following this guide, the appendix was found adherent to the under surface of the liver. The adhesions were separated with the index finger, and the appendix removed.

When the cecum is surrounded with a pus sack, we usually find a perforated and sloughing appendix, which should be removed.

In another case the writer found the appendix detached and loose in the pus sack, the stump of which was later the source of a fecal fistula, which was successfully closed by a double row of Lembert sutures.

In still another class of cases, we find a large, hard mass of infectious exudate surrounding the appendix. It is all important to break through this mass, and remove the appendix. There is little danger from hemorrhage, it seldom occurs. This done, your patient is safe. It should be a fixed rule never to close up the incision without finding and removing the appendix, 
otherwise your patient is in just as much danger as though the operation had not been performed.

Treatment of the Stump.-When the appendix is incised, the treatment of the stump resolves itself into the treatment of an intestinal perforation, which it in reality is. The surgical rules for the treatment of this condition should not be violated in treatment of the stump, viz.: Two infectious mucous surfaces should be turned in and not brought together, the object being to close the fistulous opening and prevent abdominal sepsis. Following these rules, one would suppose that all surgeons would adopt about the same method. On the contrary, Dr. Robt. H. M. Dawbarn of New York, says (May number of International Journal of Surgery, p. 139) that eleven different methods are in use by leading surgeons, all violating these rules to a greater or less degree.

The methods which have been most generally used, may be stated as follows: 1 . Incising the muscular coat, and removing it. 2. Sterilizing the stump either by Paquelin cautery, or else chemically by fuming nitric acid, or rubbing with a bichlorid of mercury tablet, or applying a drop of carbolic acid. Whichever of the foregoing processes of disinfecting may have been adopted, they have been followed by the burial of the stump beneath the peritoneum of the cecum.

While these methods are popular, they are at variance with correct surgical principles, as they leave a point of infection in the peritoneal cavity, which will sooner or later prove a serious complication. As Dawbarn wisely says: "What would be thought of a surgeon who would treat a small penetrating wound of the gut by tying a string around it, leaving two foul and infected mucous surfaces tied together?"

The various chemicals used to disinfect the stump before burying it, cause a destruction of tissue. Hence, no healing can take place between two surfaces of dead tissue.

Dr. Dawbarn's method is surgically correct, and fulfills all the conditions with the greatest safety, and is the only method which should be used, the details of which are familiar, as they have been published in the journal referred to, and in others.

Closing the Incision.- In doing this important part of the work, we are justified in assuming that the various layers of tissue are arranged in the best possible order. We will respect the work of nature by leaving them as nearly as possible as we found them. We will returm and pick up the "guy line" which we passed through the peritoneum when making the incision. By making gentle traction, the retracted peritoneum is brought into view in the upper angle of the incision. With a Martin needle loaded with No. 2 catgut, a stitch is easily passed through the free borders of the peritoneum and the edges drawn together. Remove the "guy line" and with a gentle traction on the last stitch enough of the peritoneum is drawn into the incision to easily take another stitch. This is continued until the peritoneal incision is closed by a continuous catgut suture. The transversalis fascia and transversalis and internal oblique aponeurosis is secured by a continuous suture, separately. The skin is put hard on the stretch by a tenaculum placed in the angle of the incision. The edges are united with No. 0 catgut in a fine needle, care being taken not to include any of the subcutaneous cellular tissues. This method restores the abdominal walls to their original firmness. I have not had a case of hernia following this method of closing the incision. The writer uses catgut throughout the entire operation. It is the ideal suture and ligature, if it is properly sterilized. This latter condition can only be secured by buying the raw catgut and doing your own sterilizing.

As an illustration of the advantage of the short incision, the writer gives the following case: Sept. 18, 1895 , Mrs. C. M. C. consulted me relative to a severe pain in the right iliac region. She was three and a half months pregnant, aged 25 , primipara. She gave the history of having had two distinct attacks before her marriage and pregnancy. Examination revealed great tenderness and pain over the appendix which could be distinctly felt as it was much enlarged. I advised an operation which was performed at Waternook Sanitarium, Sept. 24, 1895. The appendix was found to be much inflamed and was bound down by strong adhesions. The adhesions were broken up and the appendix removed. The stump was treated by Dawbarn's method. The incision was one and a half inches long. Patient made an excellent recovery and returned home in two weeks. I attended her at full time. She was delivered of an eight-pound daughter. The cicatrix did not yield perceptibly to the strain of pregnancy and parturition.

After-Treatment.-The patient is placed in bed, wrapped in a warm flannel blanket, with a hot waterbag at the feet. An enema of strong black coffee is administered in cases of much exhaustion. Small portions of hot water are given for the first twentyfour hours.

As we previously mentioned, we usually have an arrest of peristalsis following the operation. As a result, the bowels are greatly distended with gas, causing distressing colicky pains. Hypodermic injections of from 1-50 to 1-30 grain of strychnin are given once in four hours. This acts on the motor centers and hastens the return of peristalsis, with the result of throwing off the gas. Meanwhile, salol is administered to arrest fermentation.

When the bowel is distended for a long time the muscular coats become exhausted and the pain greatly increased. To guard against this condition, large injections of warm water are given per rectum; the gas escapes freely with the water, greatly to the relief of the patient, giving rest and relaxation to the muscular coats of the bowel. This flushing is repeated as often as it is necessary. By this treatment the colic period is shortened.

It is evident that the administration of an opiate is not a rational treatment for this condition. Opiates arrest peristalsis, the very condition we wish to overcome. Many deaths are no doubt due to the free use of opiates. A cathartic of magnesia sulphate is administered the following morning. When the bowels have moved freely, our patient goes on to a rapid convalescence.

In strongly septic cases due to pus, perforation, gangrene, etc., the wound is left wholly or partially open. A wick drainage of sterilized gauze wrapped around by gutta-percha, is inserted to the bottom of the wound. The gutta-percha covering prevents the granulations forming in the meshes of the gauze, rendering it so painful and difficult to remove. This drainage has a great advantage over the method of packing the wound with a large quantity of iodoform gauze.

The gauze, moistened with the saline solution, is 
placed over the wound. The capillary attraction wa severe, the tumor much increased in size. I then sucks up the serum, pus and other secretions through told him emphatically that I could only save his life the wick drainage, sometimes in a large quantity. A by an operation. I was then moving my private layer of cotton is placed over all, and strapped down. hospital, and had no place to take him. Dr. Chapin The gauze should be removed in about twenty-four kindly took him to a private hospital in Springfield. hours. A sterilized glass tube should be carefully May 12 the operation was performed by Dr. Chapin. inserted to the bottom of the wound, through which The incision was made over the tumor. a large it is freely irrigated with the physiologic saline solution. This may be repeated three or four times daily. Hydrogen dioxid should be used once or twice a day to disinfect the wound. Following this treatment, at the end of a week, we usually have an asceptic condition. The patient goes on to good recovery.

When perforation is present, the bowel is frequently in such a softened and diseased condition that union will not take place if the perforation is closed. Many times by waiting a few months the coats of the intestine will have regained a healthy condition, so a successful operation can be performed. In other cases, a section of the bowel is in a gangrenous condition. Only one thing remains to be done: Remove the diseased section and unite the ends with the Murphy button.

Multiple Pus Sacks and other Complications.-We occasionally meet a class of cases characterized by a high grade of infection, multiple pus sacks rapidly forming with a gangrenous perforated appendix as the center of infection. It is this class of cases which tries the skill and courage of the operator. When the abdomen is opened, a most offensive pus flows freely. The appendix is firmly bound down in the pelvic cavity by strong adhesions. Around it is built the wall of the pus sack. The infection has spread beyond this, and other pus sacks are found. It is in this class of cases that we find a great diversity of opinion as to what should be done. We will quote what Giibert Barling, M.B., F.R.C.S., Professor of Surgery in Mason's College, says (British Medical Journal, p. 1254): "When the pus is evacuated, the finger enters and searches for the appendix. It is often not recognizable, and if it is, it will almost certainly be firmly tied down by adhesions and no attempt should be made to remove it. Stripping out the appendix from the wall of the abscess is likely to break through the latter into the belly cavity, and lead to disaster. If the vermiform appendix is left, little anxiety need be felt as to future attack of appendicitis. They rarely occur."

Barling's opinion means, that little anxiety need be felt when we leave dead, decomposing, highly infectious animal tissue in the abdominal cavity. A more dangerous opinion could not be expressed.

I will relate a case which will illustrate the phase of the subject. It is one in which Dr. F. W. Chapin of Springfield, Mass., and myself were associated. At my request, Dr. Chapin operated, and I assisted him. The technique of the operation was the result of a mutual discussion of the case, and the writer assumes an equal responsibility. The lesson to be drawn from this case is too important to be omitted, as it embodied so many serious complications, viz.: pus, perforation, gangrene adhesions and secondary operation.

I first saw the patient, R. M. (a returned missionary), May 6 , at my office. He had great pain and tenderness over the appendix. I advised an immediate operation, but the patient had a volume of reasons why it should not be done. Two days later there was a well-defined tumor at the McBurney point. I urged an operation, but without avail. May 10th the pain abscess was opened and thoroughly irrigated with physiologic saline solution followed by the hydrogen dioxid. A fecal concretion was found in the pus cavity. We neither broke up the adhesions nor removed the appendix. A gauze drainage was introduced, and dressings applied. The patient recovered and was out and attended to his work. The abdominal wound closed with granulations with a small fistulous opening remaining. A few days after the operation was performed, fecal matter escaped from the wound, showing that there was a perforation.

August 5, Mr. M., the patient, came to me in Hartford, with fecal matter and pus escaping from the fistulous opening in the abdomen. I sent him at once to Waternook Sanitarium where he was properly prepared. I operated on him the next morning. I made an elliptical incision around the cicatricial tissue resulting from the first healing. I found the portion of the abdominal wall thus removed, adherent to the cecum. These adhesions were broken up revealing a perforation in the cecum. This was closed by a double row of Lembert sutures. I then broke up many strong bands of adhesions in search of the appendix, which I finally found and removed. It was in a gangrenous condition, highly infectious. I then closed up the incision, leaving the wick drainage in the lower angle of the incision, which was removed on the second day and the wound closed. Everything went well until the tenth day, when the patient became feverish, with pain near the incision. A small abscess formed external to the superficial fascia, probably from the pus germs which had burrowed in the tissues near the fistulous opening. This discharged and readily healed. At the end of four weeks, patient was up and dressed.

In reviewing this case in the light of all that happened and 'my experience with many similar cases since, I would say that at the time of the primary operation I should have thoroughly broken up all adhesions, opened and disinfected the multiple pus sacks, which existed, searched and found the appendix and removed it, as no permanent cure could be looked for with this diseased and infectious animal tissue remaining in the abdominal cavity. When at the secondary operation I had found and removed the appendix, broken up the adhesions and thoroughly disinfected the abdominal cavity, I was sure of a permanent recovery. This is what $I$ would advise in all similar cases, although eminent authorities advise to the contrary. There is a theory that the breaking up of the adhesions in pus cases, allows the pus to extravasate and infect the general peritoneal cavity. The fact is, the pus will pass out at the point of the least resistance, which is through the abdominal incision. Frequent irrigation with the use of hydrogen dioxid will render the septic condition aseptic, and a good recovery may be expected.

When the Operation Should be Performed.-In the discussion of this question, the writer will consider it from the standpoint of the best interest and safety of the patient, regardless of whims and sentimental notions.

W. Thornby Stokes, President of the Royal College 
of Surgeons in Ireland, says (British Medical Jomucl, July 1, 1895): "As far as I am able to state my opinion on the matter, it is that, if an abscess has formed or perforation taken place, laparotomy should at once be resorted to, but that, short of these misfortunes, operations should be delayed until the last possible moment."

I find in the table of statistics of operations in St. George's Hospital, in London, by T. Holmes (British Medicel Journal, July 6): Perforation of the Appendix, etc., 10 cases; recoveries 0 , deaths 10 .

This sums up President Stokes' opinion to mean: That the operation should not be performed at the time when there is a chance of saving the patient's life, but when death is inevitable, with or without operation, then "operate." The worthy president's opinion will insure a large death rate.

Now let us consider a case of a patient with the first attack of infectious appendicitis. He suffers considerable pain for three or four days, and will generally recover from this attack. If you operate, a short incision can be made. You will have no adhesions or complications. The appendix will be found to be slightly swollen and inflamed, but can be readily removed and the stump treated by Dawbarn's method, followed by little if any colic. In nine days he can sit up and in fourteen days go about his work. Now, if you slit open the appendix, you will find an inflamed spot, with probably a few drops of pus and considerable lymph thrown out. Here is the begining of an interesting and probably an eventful history.

Now, to illustrate, suppose you did not operate. In a few weeks or months he has another attack, severer and longer, resulting in some adhesions. He may go on for two years with repeated attacks, more adhesions form, an invalid condition is well established, gangrene, pus and perforation may have taken place. You then operate under the most unfavorable conditions, with a long convalescence or death. Should not operative measures be an early consid. eration, rather than the last result?

My statistics covering one hundred cases, are as follows:

Cases without extensive adhesions, infected exudate masses or pus, 27 , recoveries 27 ; cases with extensive adhesions, without infected exudate masses or pus, 25 , recoveries 25 ; cases with infected exudate masses or pus, 23, recoveries 23; cases with infected exudate masses and pus, with perforation and gangrene, 25, recoveries 23, deaths 2 ; total number of cases, 100 , recoveries 98 , deaths 2 . Males 64 , females 36 .

In conclusion, the lesson drawn from the proper appreciation of these statistics. contains an important point. We see that in classes first, second and third, no deaths occurred, but in the fourth class are two deaths. As has been stated, this class includes cases with infecterl exulate masses and pus, with perforation and gangrene. Now, with the results of an operation in this class lies the responsibility of the physician and surgeon who knowingly allows the patient to go on to the condition of this class, before an operation was performed.

\section{DISCUSSION.}

Dr. Parker, New Orleans -I have listened with much interest to the paper and congratulate the author on his brilliant results. It has been my fortune for the last seven years to be connected with the Charity Hospital of New Orleans where we have about ten thousand admissions annually and about twenty-four thousand at the out-door clinic. I have often wondered why it is that our friends in the North see so many eases of appendicitis and we so few. I do not believe we have seen half a dozen cases a year. Another peculiarity, they treat a great many negroes, but I have never seen a case of appendicitis in a negro. Dr. Johnson speaks of the catgut suture, but I believe the best suture is an absorbable one. I would like to know how he prepared this catgut. The author mentioned cases of abscess around the appendix and said he did not agree with the opinion expressed that the appendix should not be removed in these cases. In several of my cases, there were abscesses around the appendix and in some cases, the appendix was in the abscess wall. I believe that if we had started in to remove those appendices we would have opened the general peritoneal cavity. Instead of this we opened the abscesses and washed out the region with peroxid, all of the cases getting well and in not one of them has there been a recurrent attack. As to the use of opiates and salines, I believe in the way of preparatory treatment that it is a good plan to open the bowel with an enema and saline. We are all pretty well agreed as to the method of operating, but I do not believe in the use of opium. With regard to fecal fistulae, I have seen several cases. Some of them will heal up by themselves if you let them alone.

ThE SECRETARY-The results secured by the author of the paper are almost unparalleled, and such statements coming from a man of such experience and with such good fortune or rather good tcchnique must have a strong impression. Statements read from such a paper would probably be carried away from this section as the authoritative statements of this section and I feel they should be discussed. Another point, that of searching for the appendix in cases of abscess in every in stance. I think this matter has been gone into by some of the best authorities. Having been a pupil of old Dr. Henry Parker, who was the pioneer in the advocation of opening appendiceal abscesses, I saw many abscesses which were undoubtedly what we would have called at that time perityphlitic, and I watched some of these cases for years. I do not remember of a single recurrence of appendicitis after the abscesses were opened and drained. It is a dangerous doctrine to set down any definite technique for any operation, and every case must be judged on its own merits. You might as well say treat all cases of pneumonia with the same drug. The external incision in some cases may be one inch, in others, two inches and sometimes from seven to eight inches, and this must be decided in every individual case according to the requirements of that case. Dr. Dawbarn's recommendation is an excellent one, but there are cases in which it is impracticable to use it. I recently had a case of recurrent appendicitis and I operated during the third attack. I found the appendix as large and hard as my little finger and it was perfectly indurated. The whole lumen of the appendix was destroyed and seemed to be replaced by an enormously indurated wall which was like a hard piece of rigid tube. It was thoroughly impracticable to have cut off that appendix and in such cases we must be guided by the case and not by any definite technique.

Dr. Carpenter, Kentucky-Each case is a law unto itself and must be treated in that way. As to suppurative appendicitis, we can not afford to tear up adhesions and run the risk of opening the peritoneal cavity and poisoning it. If we excise, irrigate and drain; we will save the case as a rule and I think many a patient is lost by meddlesome surgery. I recently operated on a patient in which the appendix was gangrenous and half sloughed off from the cecum. The cecum was gangrenous for about two inches and the appendix contained an appendolith. I removed this and cut off the gangrenous edges of the cecum. I employed the Lembert suture. I took the meso-appendix and brought it over, drained, irrigated and the patient recovered.

DR. MCRAE, Atlanta-I have only had a few cases. One of the points raised by Dr. Parker, I can answer in the affirmative, and that is that I have operated on two cases of appendicitis in the negro, one male and one femaie. It is true they had a little white blood in them, but they were negroes all the same. They both recovered. I believe that it would be one of the worst theories or practices to state that every appendix should be removed when it is contained in the abscess wall, and 1 am firmly convinced that it would be a very dangerous practice. I have read the works of many of the English surgeons and the universal experience seems to be that where there is a large abscess cavity with the appendix contained in the walls, simply draining is all that is necessary. It is the exception and not the rule for recurrence to take place. As Dr. Joseph Price has said, when you have a condition of that kind, the time for ideal surgery is not present and you simply wish to conserve the life of the patient. If it becomes necessary to remove the appendix later, I am satisfied that it could be done with less danger than by doing it at one sitting. A large proportion of those cases will die from septic peritonitis. I have had two cases of fecal 
fistulae where the abscesses were drained after removal of appendix and one case in which I drained the abscess. If we let them alone, they will get well.

Dr. Alex. Hugh Ferguson, of Chicago-I can not refrain from commenting on the surgical manner in which Dr. Parker made his remarks. I should like to be excused from discussing the paper as presented to us, but there are a number of points presented which to my mind are not the best procedures and I would like therefore to give a brief outline of how I manage cases myself. I have only had an experience of about 150 cases. I am surprised at the few cases that occur in the South and I think it must be due to the climate. The medical pictures that present themselves to us are, you might say, acute cases, and demand immediate operation. There are some cases which ars not so severe, which recover and then have secondary attacks, and then there are others which return after operation when they have simply been drained. I have only had two cases of this myself. They were facsimilies of Dr. McRae's case where the appendix proper was obliterated, but there was a chronic abscess which would become full and cause an attack simulating appendicitis. The most favorable time to operate is between the attacks, and that old surgical rule is good. It is dangerous to operate during the acme of inflammation but sometimes we have to operate at this time, particularly whon there is a tumor, otherwise we do not know whether it may or may not burst. The second most favorable time to operate is early, as soon as we have a clear clinical picture of a case of appendicitis, where it is evident that the inflammation is around the appendix. Early operation, say within twenty-four hours, is much more easily done, and the patient is much safer than if you leave it until the fourth or fifth day. The technique of operation varies. An inch and a half and a week and a half is wrong in 99 per cent. of the cases. You may do such an operation between the attacks where the intlammatory process is limited to the inside of the appendix. I once saw a surgeon who was fond of using that old expression, "an inch and a half and a week and a half," who went into the general peritoneal cavity by mistake. The technique of an operation between the attacks is clear. Select your incision and separate the fibers of the muscles. You then expose the appendix that has been inflamed in its quiescent state, and mike a circular incision, tying off the mucous membrane, or as I prefer, inverting it by a figure of 8 . A simple ligature over the muscular walls is the proper method to my mind. You can not always get your stump in good condition for this treatment but you should drain anyway. If you have to search for the appendix and break down adhesions, it is not necessary to drain. When there is no way of removing the appendix, as in appendicitis fibrosa or where we have much traumatism, capillary drainage is all that is needed. This will drain blood serum but not pus and that point should be looked after. We never aspirate unless the appendix is situated in a region which can be quite easily gotten at. There is no adran tage in removing the appendix through the peritoneal cavity, and it is a very difficult way, but it should not be allowed to slough off. When it is in the pelvis, it should not be hunted for. Just as soon as you strike pus, drain it or mop it out In draining, do so with gauze and with a tube, as gauze will not alone drain pus. The best drain with the gauze is a glass drainage tube, as a rubber one may become clogged up or may be pressed upon by the gas present. I was recently called to the case of a young man who had been riding a bicycle, and a few hours after having been thrown off, he complained of pain in the right iliac region. A physician had been called who diagnosed the case one of appendicitis. I was called about twenty-eight hours later, when the patient had a temperature of 103 and pulse 120 . The abdomen was not very much distended but the muscles were rigid. An operation was agreed to and I cut down and removed the appendix, afterward packing with gauze and draining with a glass tube.

Dr. Haines, Omaha-I would like to congratulate the author upon his good paper. The first case I had was my most difficult one, and I found extensive adhesions in the right iliac fossa. After a long search I discovered quite a mass, and I felt the appendix within it. My case went the way of all flesh, but $I$ believe if $I$ had had the experience I now have, he would still be in the land of the living. I do not mean to say that it is always necessary to remove the appendix. It has been suggested to treat it as an abscess, which may be a good method. I live in an atmosphere that is impregnated with appendicitis, and we hear of dozens of cases in our local society every time we meet. It occurs in both races. I merely wish to condemn the routine practice of always searching for the appendix.

Dr. Johnson (in closing the discussion)-I expected a dis cussion and am pleased to have gotten it. Perhaps I hold a unique position in this operation, as I have been both operator and patient. There is one thing about searching for the appendix. I have done this in the presence of as good surgeons as are here to-day and they have often warned me to discon tinue my search. Where you have one large pus sac, you may drain, but almost invariably you will have multiple pus sacs. I find by breaking up the adhesions that you will find you will break into a pus sac just as you may open a wound. I have found as many as five pus sacs in one case, and if you leave these sacs I think they will do harm. Perhaps if some of you will just try the method of breaking up the wall of a pus sac after you have broken up several, you will probably find others near by which will cause, upon rupture, quite a flow of pus. These sacs exist to a large extent, and have been found in the liver and in the whole abdominal cavity. Some patients have died without operation. When the appendix is situated, as it almost always is, in the wall of the pus sac, I remove the ppendix. I am aware that you can mention good authority against this procedure, but my experience is that the pus will extravasate into the abdominal cavity and not come out through the incision. From my method I have never had a case of peritonitis resulting, and I think my procerture the proper one. You have a highly septic vegetable matter which it is dangerous to leave behind although I did not think so until I had passed a case where the appendix was removed in a secondary operation. Up to the present time $I$ have had about 125 cases. I think $I$ have seen a great many patients that would not have recovered merely by breaking up the pus sacs. I was sorry to note that Dr. Ferguson did not give any statistics. I would like to ask some of you to try my method. I have tried it although many men have told me not to do it. As to the length of the incision, I think it is often made too long, and a seven-inch incision should not be required in any case. It not only prolongs the operation, but there will certainly be an escape of the small intestines. My experience is that in most complicated cases an incision two or two and a half inches long is enough, and is sufficient to break up adhesions by the sense of touch. A surgeon once saw me operate and remove the appendix, and said he was unable to see or understand how I did it. I think the best way is to enlarge the incision if necessary and that in so doing you are considering the welfare of your patient. When you make a long incision a weakened abdominal wall is the result, and if the patient be a laboring man a strong abdominal wall is of great service to him. Experience soon enables one to perform this operation through a very small incision. If any of you will try this method, you will find that your result will juetify you in doing it. The free use of boracic acid and hydrogen will save many cases, and I have been perfectly satisfied with my results. Physicians do wrong in allowing cases to go on before sending for a surgeon. The Dawbarn method can not be performed in every case, but where it can be, I think it should be as there are no infectious points left free in the abdominal cavity. When you first begin to employ this method, you will probably think you will not succeed, but it can be done in five minutes after you get used to it. With regard to fecal fistula, this will usually take care of itself. I remember one case in which the cecum was so far gone that it required a second operation. With regard to the employment of catgut, $\boldsymbol{i}$ buy it in the raw state and sterilize it my self. I first place it in ether and continue to change it until all the fat is taken out of it. I then boil it at a temperature of 173 , and then put it away in sterilized glass jars. When I come to operate, I take out the size and quantity I want and again sterilize it. I have so far had no cause to regret its use.

\section{A FEW RECENT CASES BEARING UPON THE QUESTION OF OPERATIVE INTER-} FERENCE IN ABDOMINAL AILMENTS.

Read in the Section on Surgery aud Anatomy at the Forty. seventh Annual Meeting of the American Medical Association held at

BY DONALD MACLEAN, M.D., LL.D. DETROIT, MICH.

In the following brief paper my main object is of furnish the text for a discussion, by this Section, to one of the most anxious and difficult problems in practical surgery, namely the question of interference or non-interference in cases of abdominal ailment, traumatic or otherwise 